\title{
Institutional Quality and Economic Growth: The Case of Emerging Economies
}

\author{
Canh Phuc Nguyen1, Thanh Dinh Su², Thai Vu Hong Nguyen ${ }^{3}$ \\ ${ }^{1}$ School of Banking, University of Economics Ho Chi Minh City, Vietnam \\ ${ }^{2}$ School of Public Finance, University of Economics Ho Chi Minh City, Vietnam \\ ${ }^{3}$ School of Business and Management, RMIT Vietnam, Ho Chi Minh City, Vietnam \\ Email: ngvh.thai@gmail.com
}

How to cite this paper: Nguyen, C.P., $\mathrm{Su}$ T.D. and Nguyen, T.V.H. (2018) Institutional Quality and Economic Growth: The Case of Emerging Economies. Theoretical Economics Letters, 8, 1943-1956. https://doi.org/10.4236/tel.2018.811127

Received: May 7, 2018

Accepted: July 20, 2018

Published: July 23, 2018

Copyright (c) 2018 by authors and Scientific Research Publishing Inc. This work is licensed under the Creative Commons Attribution International License (CC BY 4.0).

http://creativecommons.org/licenses/by/4.0/

\begin{abstract}
The roles of institutional quality on economic growth are still heavily debated in the literature. This paper investigates the impacts of institutional quality on economic growth for 29 emerging economies over the 2002-2015 period by employing System Generalized Method of Moments (SGMM) estimators. We find the significant positive impacts of institutional quality on economic growth. The institutional quality impedes the positive effects of foreign direct investments (FDIs) and trade openness on economic growth. However, institutional quality improvement can mitigate the competition brought by trade openness in the areas FDIs operate to optimize their spill-over effect.
\end{abstract}

\section{Keywords}

Institutional Quality, Economic Growth, Emerging Economies, System GMM

\section{Introduction}

An important issue for both academic and pratical studies is the extent to which determinants of economic growth play in different countries [1], meanwhile the economic grow this essential to maintain and improve the international competiveness of a country [2] [3]. Although economic growth has been widely researched, the traditional economic theories lack a framework explaining the differences in economic systems between countries beyond human capital, physical capital, labor, technology, and natural resources (e.g., see [4] [5]). Recent research in institutional economics has arisen as an attempt to provide a framework investigating those residual differences ([6] [7]). High institutional quality has been argued as an economic growth momentum by incentivizing economic activities such as consumption and investment [8] [9], improving effi- 
ciency [10], allocating resources more efficiently [11] [12], protecting property rights and supporting freedom of choice [13].

The literature also argues the indirect impact of institutional quality on Gross Domestic Product (GDP) via the growth effect of trade openness [14] and the growth effect of Foreign Direct Investment (FDI) [15]. Institutional quality has been found to strengthen the economic growth effect of trade openness as better institutional quality tends to accelerate the advantages from trade such as specialization and economies of scale in advanced economies [14]. Nevertheless, emerging economies are less comparative and trade advantages may take time to materialize [16]. This raises the concern on the impact of institutional quality improvement on the economic growth effect of trade openness in emerging economies. In addition, the literature largely supports that good institutional quality can escalate the economic growth effect of FDI by better facilitating the technology transfer and knowledge spill-over processes [17]. However, the literature largely ignores the impact of institutional quality on the economic growth effects of both trade openness and FDI simultaneously. This study is the first attempt examining the role of institutional quality in moderating the impacts of the nexus of trade openness and FDI on economic growth in emerging economies. This is imperative as emerging economies are increasingly opening to trade and foreign investment which may mutually affect economic growth.

This study contributes to the literature on two fronts. First, the study provides new arguments on the impact of institutional quality on the economic growth effect of trade openness in the short run for emerging economies. In contrast to the evidence documented for developed countries, institutional quality improvement tends to impede the economic growth effect of trade openness and FDI in emerging countries in the short run. Second, the study extends the economic growth theories to incorporate the role of institutional quality on the mutual effects of trade and FDI on economic growth in emerging economies. The study finds that better institutional quality can mitigate the competition brought by trade openness in the areas that FDIs operate to optimize their spillover effect. The remaining of the study is organized as followings. Section two reviews the literature and develops hypotheses. Section three discusses data and methodology. Section four presents the data analysis. Section five concludes the study.

\section{Literature Review and Hypothesis Development}

\subsection{Institutional Quality and Growth Effect of Trade Openness}

The impact of trade openness on economic growth has been well studied following endogenous growth model (see [18] [19]). This strand of literature argues that trade openness tends to enhance economic growth by improving domestic productivity and taking advantage of economies of scale which encourages specialization. This is because trade openness stimulates competition which pres- 
sures domestic firms to innovate for higher production efficiency (see [14]). Competition also serves as the channel to eliminate uncompetitive firms, so that resources will be allocated to more competitive firms which further improve economic growth [14]. The empirical studies largely support the positive effect of trade openness to economic growth (for example, [20]). The literature also argues the important role of institutional quality on the growth effect of trade openness (see [14] for the review). The literature points out that better institutional quality could accelerate the growth effect of trade openness by better facilitating efficiency improvement process and strengthening the advantages of economies of scale [14] [16]. Our study argues that, in the short run, better institutional quality may impede the growth effect of trade openness. We argue that better institutional quality reduces uncertainties related to international trade activities such as imperfect contract enforcement [21], and protection of intellectual property rights [22], competition brought by foreign firms becomes fiercer. While it takes time for domestic firms to promote efficiency, fierce competition may force domestic firms to reduce their production as the market is shared to foreign counterparts. If the domestic market share shrink is not compensated by exporting expansion, better institutional quality reduces the growth effect of trade openness. In addition, it has been documented that emerging economies are not as efficient as advanced economies [23], hence, better institutional quality may facilitate import more than export for emerging economies, leading to slower economic growth in the short run. For this reason, we hypothesize that the interaction of institutional quality and trade openness has a negative impact on economic growth in emerging economies (Hypothesis 1).

\subsection{Institutional Quality and Growth Effect of FDI}

Endogenous growth theory posits that the FDI spillover effect (i.e. technology transfer and knowledge spillover) can be transformed into productivity improvement and consequently economic growth [24] [25]. In addition, FDI also complements to domestic investment by relaxing the liquidity constraint, therefore, escalating economic growth [26]. [17] reviews the empirical studies on the impact of FDI on economic growth and points out that the mixed evidence is conditional on the institutional quality. [17] argues that poor institutional quality is associated with high transaction costs, increased risk for long term commitment, loosening the linkages between foreign affiliates and domestic firms, and hence, limits the spillover effect. In addition, better institutional quality is also argued to mitigate the crowding out effect of FDI by encouraging foreign investment into pioneering industries and lessening competition with domestic firms, so that the growth effect of FDI will be further strengthened. In line with this argument, we posit that the positive effect of FDI on economic growth will be stronger in countries with relatively high institutional quality. We further examine the impact of the nexus of institutional quality, FDI and trade openness on economic growth. 


\section{Methodologies and Data}

The study investigates both the direct effects of institutional quality on economic growth and the indirect effects of institutional quality on economic growth through the interactions with the international trade and capital flows in emerging markets. Following [27], the impact of institutional quality is estimated in the model as below:

$$
\begin{aligned}
y_{i t}= & \alpha_{1} y_{i t-1}+\beta_{j} X_{j, i t}+\partial_{1} \text { Trade }_{i t}+\partial_{2} \mathrm{FDI}_{i t}+\partial_{3} \text { Inst }_{i t} \\
& +\partial_{4}(\text { Trade } * \mathrm{FDI})_{i t}+\partial_{5}(\text { Inst } * \text { Trade })_{i t}+\partial_{6}(\text { Inst } * \mathrm{FDI})_{i t} \\
& +\partial_{7}(\text { Inst } * \text { Trade } * \mathrm{FDI})_{i t}+\varepsilon_{i t}
\end{aligned}
$$

The economic growth $(y)$ is proxied by the growth rate of real GDP (Gdpg) and the growth rate of real GDP per capita (Gdppcg). Trade openness (Trade) is measured as the total import and export value per GDP. FDI is measured as the ratio of net inflows foreign direct investment per GDP.

The vector of control variables $(X)$ in this estimation includes the logarithm of real GDP per capita ( $\operatorname{Logg} d p p c)$, the growth rate of gross capital formation (Capg), the logarithm of total non-resident and resident patent applications (Tech), population growth (Popg) (for the equation of GDP growth rate as dependent variable, it is excluded in the case of GDP per capita growth rate since the dependent variable has reflected the dynamic in both output and population), and logarithms of ratio of tertiary school enrollment to population (Humcap). These variables are used to control for level of economic development, capital factor, technology change, labor and human capital, respectively [28] [29] [30].

The institutional quality (inst) is proxied alternatively by the percentage change in government effectiveness index (Goveff), and the percentage change in regulatory quality index (Requa), control of corruption (Concor), political stability (Politic), rule of law (Rulelaw), and voice and accountability (Voice). These are extracted from World Governance Indicators (WGI) and range from approximately -2.5 (weak) to 2.5 (strong) governance performance), and higher estimated values represents better institutional quality. Despite there are arguments for the reliability of these indicators for institutional quality, many previous studies have used and documented that they are our best choice until now (see [9] [31]).

The yearly data on institutional quality is collected from World Governance Indicators to the latest available time with continuous data from 2002 to 2015 for 29 emerging countries in Appendix 1. Other variables are collected from the World Development Index. All the definitions and calculations are presented in Appendix 2. The data description is presented in Table 1.

\section{Results and Discussions}

The model is estimated with SGMM estimator to control for potential endonegeity ([32] [33]). This estimator is also argued to be efficient for unbalanced 
Table 1. Data description.

\begin{tabular}{cccccc}
\hline Variables & Obs. & Mean & Std. Dev. & Min & Max \\
\hline Gdpg & 405 & 4.112 & 3.685 & -14.800 & 14.231 \\
Gdppcg & 405 & 3.273 & 3.601 & -14.421 & 13.636 \\
Capg & 405 & 5.302 & 12.554 & -57.400 & 67.389 \\
Tech & 396 & 8.285 & 1.712 & 5.455 & 13.913 \\
Loggdppc & 405 & 8.718 & 1.004 & 6.285 & 10.408 \\
Popg & 406 & 0.817 & 0.843 & -2.171 & 2.390 \\
Humcap & 367 & 3.663 & 0.684 & 1.004 & 4.735 \\
Trade & 405 & 4.202 & 0.517 & 3.096 & 5.349 \\
Fdi & 405 & 3.382 & 4.536 & -16.071 & 50.742 \\
Concor & 406 & -0.177 & 0.613 & -1.488 & 1.573 \\
Goveff & 406 & 0.160 & 0.610 & -0.889 & 1.375 \\
Politic & 406 & -0.433 & 0.895 & -2.806 & 1.202 \\
Requa & 406 & 0.150 & 0.720 & -1.730 & 1.547 \\
Rulelaw & 406 & -0.052 & 0.677 & -1.033 & 1.419 \\
Voice & 406 & 0.005 & 0.791 & -1.687 & 1.244 \\
\hline & & & & & \\
\hline
\end{tabular}

data with relatively small time period and large cross-section groups (countries) ([32] [33]).

Table 2 and Table 3 present the baseline results without institutions for GDP per capita growth and GDP growth, respectively. The error terms are free of unit root and serial correlation. Population growth is included in GDP growth regression only. Capital growth, technology, population growth, FDI and trade openness significantly improve economic growth. The positive impact of human capital is only statistically significant in GDP per capita growth, but not in GDP growth. Economic growth tends to slow down at higher GDP per capita levels. This confirms the converging trend in economic growth among emerging markets, and this is consistent with the converging theory in economic growth field [34] [35].

The impacts of institutional quality on GDP per capita and GDP growth are presented in Table 4 and Table 5, where the role of each institutional quality component is examined alternatively. The results in Table 4 show that control of corruption, government effectiveness, rule of law, and voice and accountability significant improve GDP per capita growth. These results are in line with theories and previous studies on the effects of institutional quality on economic growth [36] [37]. Although political stability and regulatory quality have expected positive impacts on GDP per capita growth, they are not statistically significant. All six elements of institutional quality do not show any significant impacts on GDP growth in Table 5.

Table 6 and Table 7 show the impacts of institutions in conjunction with FDI 
Table 2. GDP per capita growth.

\begin{tabular}{cccc}
\hline Dep: GDP per capita growth & $(1)$ & $(2)$ & $(3)$ \\
\hline L1. GDPpcg & $0.3203^{* * *}$ & $0.2744^{* * *}$ & $0.2138^{* * *}$ \\
& {$[0.0334]$} & {$[0.0421]$} & {$[0.0446]$} \\
Loggdppc & $-0.3412^{* * *}$ & $-0.5045^{* * *}$ & $-0.6304^{* * *}$ \\
Capg & {$[0.0870]$} & {$[0.1014]$} & {$[0.1021]$} \\
Tech & $0.2600^{* * *}$ & $0.2487^{* * *}$ & $0.2425^{* * *}$ \\
& {$[0.0102]$} & {$[0.0092]$} & {$[0.0067]$} \\
Humcap & $0.2012^{* * *}$ & $0.2483^{* * *}$ & $0.2391^{* * *}$ \\
FDI & {$[0.0340]$} & {$[0.0437]$} & {$[0.0400]$} \\
Trade & $0.5851^{* * *}$ & $0.8106^{* * *}$ & $0.5937^{* * *}$ \\
N & {$[0.1625]$} & {$[0.1625]$} & {$[0.1563]$} \\
No. of Group & & $0.0549^{* *}$ & $0.0703^{* *}$ \\
No. of IVs & & {$[0.0226]$} & {$[0.0318]$} \\
AR(2) test & 315 & & $0.5277^{* * *}$ \\
Sargan test & 29 & 261 & {$[0.1106]$} \\
Hansen test & 26 & 29 & 260 \\
\hline
\end{tabular}

Notes: $\left(^{*}\right),\left({ }^{* *}\right),\left({ }^{* *}\right)$ are significant at $10 \%, 5 \%$, and $1 \%$ level respectively.

Table 3. GDP growth.

\begin{tabular}{|c|c|c|c|}
\hline Dep: GDP growth & (4) & (5) & (6) \\
\hline L1. GDPg & $\begin{array}{c}0.3018^{* * *} \\
{[0.0246]}\end{array}$ & $\begin{array}{c}0.2717^{\star * *} \\
{[0.0368]}\end{array}$ & $\begin{array}{c}0.2433^{* * *} \\
{[0.0421]}\end{array}$ \\
\hline Loggdppc & $\begin{array}{l}-0.1288 \\
{[0.0963]}\end{array}$ & $\begin{array}{l}-0.1278 \\
{[0.0801]}\end{array}$ & $\begin{array}{c}-0.4348^{* * *} \\
{[0.1017]}\end{array}$ \\
\hline Capg & $\begin{array}{c}0.2591^{* * *} \\
{[0.0102]}\end{array}$ & $\begin{array}{c}0.2332^{* * *} \\
{[0.0087]}\end{array}$ & $\begin{array}{c}0.2457^{* * *} \\
{[0.0099]}\end{array}$ \\
\hline Tech & $\begin{array}{c}0.2679^{* * *} \\
{[0.0247]}\end{array}$ & $\begin{array}{c}0.2788^{* * *} \\
{[0.0336]}\end{array}$ & $\begin{array}{c}0.3040^{* * *} \\
{[0.0388]}\end{array}$ \\
\hline Humcap & $\begin{array}{c}0.0578 \\
{[0.1962]}\end{array}$ & $\begin{array}{c}0.0003 \\
{[0.1713]}\end{array}$ & $\begin{array}{c}0.0198 \\
{[0.1618]}\end{array}$ \\
\hline Popg & $\begin{array}{c}0.2801^{\star * *} \\
{[0.0813]}\end{array}$ & $\begin{array}{c}0.3742^{\star * *} \\
{[0.0952]}\end{array}$ & $\begin{array}{c}0.3590^{* * *} \\
{[0.1257]}\end{array}$ \\
\hline FDI & & $\begin{array}{l}0.0825^{*} \\
{[0.0420]}\end{array}$ & $\begin{array}{l}0.0764^{* *} \\
{[0.0343]}\end{array}$ \\
\hline Trade & & & $\begin{array}{c}0.5397^{* * *} \\
{[0.0997]}\end{array}$ \\
\hline $\mathrm{N}$ & 315 & 287 & 261 \\
\hline No. of Group & 29 & 29 & 29 \\
\hline No. of IVs & 27 & 28 & 28 \\
\hline $\operatorname{AR}(2)$ test & 0.734 & 0.803 & 0.848 \\
\hline Sargan test & 0.369 & 0.163 & 0.494 \\
\hline Hansen test & 0.491 & 0.579 & 0.413 \\
\hline
\end{tabular}

Notes: $\left(^{*}\right),\left({ }^{*}\right)$, and $\left({ }^{* *}\right)$ are significant at $10 \%, 5 \%$, and $1 \%$ level respectively. 
Table 4. Institutional quality and GDP per capita growth.

\begin{tabular}{|c|c|c|c|c|c|c|}
\hline $\begin{array}{l}\text { Dep: GDP per } \\
\text { capita growth }\end{array}$ & $\begin{array}{l}\quad(7) \\
\text { Control of } \\
\text { corruption } \\
\text { (Concor) }\end{array}$ & $\begin{array}{c}\text { (8) } \\
\text { Government } \\
\text { effectiveness } \\
\text { (Goveff) }\end{array}$ & $\begin{array}{c}\text { (9) } \\
\text { Political } \\
\text { stability } \\
\text { (Politic) }\end{array}$ & $\begin{array}{c}(10) \\
\text { Regulatory } \\
\text { quality } \\
\text { (Requa) }\end{array}$ & $\begin{array}{c}(11) \\
\text { Rule of Law } \\
\text { (Rulelaw) }\end{array}$ & $\begin{array}{c}(12) \\
\text { Voice \& } \\
\text { Accountability } \\
\text { (Voice) }\end{array}$ \\
\hline L1. GDPpcg & $\begin{array}{c}0.2076^{* * *} \\
{[0.0535]}\end{array}$ & $\begin{array}{c}0.2062^{* * *} \\
{[0.0531]}\end{array}$ & $\begin{array}{c}0.1993^{* * *} \\
{[0.0529]}\end{array}$ & $\begin{array}{c}0.2061^{* * *} \\
{[0.0523]}\end{array}$ & $\begin{array}{c}0.2016^{* * *} \\
{[0.0538]}\end{array}$ & $\begin{array}{c}0.1993^{* * *} \\
{[0.0519]}\end{array}$ \\
\hline Loggdppc & $\begin{array}{c}-0.8832^{* * *} \\
{[0.1187]}\end{array}$ & $\begin{array}{c}-0.8798^{* * *} \\
{[0.1081]}\end{array}$ & $\begin{array}{c}-0.8106^{* * *} \\
{[0.0969]}\end{array}$ & $\begin{array}{c}-0.9040^{* * *} \\
{[0.1201]}\end{array}$ & $\begin{array}{c}-0.8734^{* * *} \\
{[0.1050]}\end{array}$ & $\begin{array}{c}-0.8746^{* * *} \\
{[0.0912]}\end{array}$ \\
\hline Capg & $\begin{array}{c}0.2482^{\star * *} \\
{[0.0094]}\end{array}$ & $\begin{array}{c}0.2486^{* * *} \\
{[0.0096]}\end{array}$ & $\begin{array}{c}0.2467^{\star * *} \\
{[0.0094]}\end{array}$ & $\begin{array}{c}0.2488^{* * *} \\
{[0.0095]}\end{array}$ & $\begin{array}{c}0.2498^{* * *} \\
{[0.0096]}\end{array}$ & $\begin{array}{c}0.2456^{* * *} \\
{[0.0098]}\end{array}$ \\
\hline Tech & $\begin{array}{c}0.3239^{* * *} \\
{[0.0598]}\end{array}$ & $\begin{array}{c}0.3166^{* * *} \\
{[0.0491]}\end{array}$ & $\begin{array}{c}0.3128^{* * *} \\
{[0.0595]}\end{array}$ & $\begin{array}{c}0.3351^{* * *} \\
{[0.0605]}\end{array}$ & $\begin{array}{c}0.3266^{* * *} \\
{[0.0515]}\end{array}$ & $\begin{array}{c}0.3449^{* * *} \\
{[0.0441]}\end{array}$ \\
\hline Humcap & $\begin{array}{c}0.7790^{\star * \star} \\
{[0.1767]}\end{array}$ & $\begin{array}{c}0.8082^{\star * \star} \\
{[0.1794]}\end{array}$ & $\begin{array}{c}0.7446^{\star * \star} \\
{[0.1886]}\end{array}$ & $\begin{array}{c}0.8310^{\star * *} \\
{[0.1776]}\end{array}$ & $\begin{array}{c}0.7914^{\star * *} \\
{[0.1722]}\end{array}$ & $\begin{array}{c}0.7379^{* * *} \\
{[0.1970]}\end{array}$ \\
\hline FDI & $\begin{array}{c}0.0683^{*} \\
{[0.0386]}\end{array}$ & $\begin{array}{c}0.0672^{*} \\
{[0.0393]}\end{array}$ & $\begin{array}{c}0.0796^{*} \\
{[0.0391]}\end{array}$ & $\begin{array}{c}0.0784^{*} \\
{[0.0403]}\end{array}$ & $\begin{array}{l}0.0720^{*} \\
{[0.0390]}\end{array}$ & $\begin{array}{l}0.0823^{* *} \\
{[0.0400]}\end{array}$ \\
\hline Trade & $\begin{array}{c}0.7365^{* * *} \\
{[0.0949]}\end{array}$ & $\begin{array}{c}0.6933^{* * *} \\
{[0.1030]}\end{array}$ & $\begin{array}{c}0.6372^{\star * *} \\
{[0.1275}\end{array}$ & $\begin{array}{c}0.6829^{* * *} \\
{[0.1047]}\end{array}$ & $\begin{array}{c}0.6879^{* * *} \\
{[0.1092]}\end{array}$ & $\begin{array}{c}0.7012^{* * *} \\
{[0.1118]}\end{array}$ \\
\hline Inst & $\begin{array}{l}0.3291^{* *} \\
{[0.1481]}\end{array}$ & $\begin{array}{l}0.3153^{* *} \\
{[0.1399]}\end{array}$ & $\begin{array}{c}0.0896 \\
{[0.1278]}\end{array}$ & $\begin{array}{c}0.2092 \\
{[0.1440]}\end{array}$ & $\begin{array}{l}0.2978^{* *} \\
{[0.1342]}\end{array}$ & $\begin{array}{l}0.2171^{\star *} \\
{[0.0997]}\end{array}$ \\
\hline $\mathrm{N}$ & 232 & 232 & 232 & 232 & 232 & 232 \\
\hline No. of Group & 29 & 29 & 29 & 29 & 29 & 29 \\
\hline No. of IVs & 26 & 26 & 26 & 26 & 26 & 26 \\
\hline $\mathrm{AR}(2)$ test & 0.797 & 0.803 & 0.829 & 0.813 & 0.792 & 0.838 \\
\hline Sargan test & 0.147 & 0.153 & 0.135 & 0.147 & 0.147 & 0.130 \\
\hline Hansen test & 0.621 & 0.638 & 0.632 & 0.616 & 0.648 & 0.665 \\
\hline
\end{tabular}

Notes: $\left(^{*}\right),\left(^{* *}\right),\left({ }^{* *}\right)$ are significant at $10 \%, 5 \%$, and $1 \%$ level respectively.

Table 5. Institutional quality and GDP growth.

\begin{tabular}{|c|c|c|c|c|c|c|}
\hline $\begin{array}{l}\text { Dep: GDP } \\
\text { growth }\end{array}$ & $\begin{array}{l}\text { (13) } \\
\text { Control of } \\
\text { corruption } \\
\text { (Concor) }\end{array}$ & $\begin{array}{c}\text { (14) } \\
\text { Government } \\
\text { effectiveness } \\
\text { (Goveff) }\end{array}$ & $\begin{array}{c}(15) \\
\text { Political } \\
\text { stability } \\
\text { (Politic) }\end{array}$ & $\begin{array}{c}\text { (16) } \\
\text { Regulatory } \\
\text { quality } \\
\text { (Requa) }\end{array}$ & $\begin{array}{c}(17) \\
\text { Rule of Law } \\
\text { (Rulelaw) }\end{array}$ & $\begin{array}{c}(18) \\
\text { Voice \& } \\
\text { Accountability } \\
\text { (Voice) }\end{array}$ \\
\hline L1. GDPg & $\begin{array}{c}0.2360^{* * *} \\
{[0.0430]}\end{array}$ & $\begin{array}{c}0.2422^{\star * *} \\
{[0.0431]}\end{array}$ & $\begin{array}{c}0.2376^{\star * *} \\
{[0.0432]}\end{array}$ & $\begin{array}{c}0.2402^{\star * *} \\
{[0.0440]}\end{array}$ & $\begin{array}{c}0.2379^{\star * *} \\
{[0.0425]}\end{array}$ & $\begin{array}{c}0.2431^{\star * *} \\
{[0.0418]}\end{array}$ \\
\hline Loggdppc & $\begin{array}{c}-0.4659^{* * *} \\
{[0.1185]}\end{array}$ & $\begin{array}{c}-0.4672^{* * *} \\
{[0.1147]}\end{array}$ & $\begin{array}{c}-0.4491^{* * *} \\
{[0.1169]}\end{array}$ & $\begin{array}{c}-0.4779^{* * *} \\
{[0.1256]}\end{array}$ & $\begin{array}{c}-0.4602^{* * *} \\
{[0.1114]}\end{array}$ & $\begin{array}{c}-0.4414^{* * *} \\
{[0.0972]}\end{array}$ \\
\hline Capg & $\begin{array}{c}0.2514^{* * *} \\
{[0.0085]}\end{array}$ & $\begin{array}{c}0.2514^{* * *} \\
{[0.0083]}\end{array}$ & $\begin{array}{c}0.2485^{* * *} \\
{[0.0091]}\end{array}$ & $\begin{array}{c}0.2494^{* * *} \\
{[0.0087]}\end{array}$ & $\begin{array}{c}0.2506^{* * *} \\
{[0.0087]}\end{array}$ & $\begin{array}{c}0.2442^{* * *} \\
{[0.0103]}\end{array}$ \\
\hline Tech & $\begin{array}{c}0.2877^{* * *} \\
{[0.0436]}\end{array}$ & $\begin{array}{c}0.2853^{\star * *} \\
{[0.0412]}\end{array}$ & $\begin{array}{c}0.3006^{* * *} \\
{[0.0479]}\end{array}$ & $\begin{array}{c}0.2985^{\star * *} \\
{[0.0429]}\end{array}$ & $\begin{array}{c}0.2914^{\star * *} \\
{[0.0427]}\end{array}$ & $\begin{array}{c}0.3171^{\star * *} \\
{[0.0403]}\end{array}$ \\
\hline Humcap & $\begin{array}{c}0.0831 \\
{[0.1956]}\end{array}$ & $\begin{array}{c}0.0586 \\
{[0.1850]}\end{array}$ & $\begin{array}{c}0.0536 \\
{[0.1965]}\end{array}$ & $\begin{array}{c}0.0618 \\
{[0.1992]}\end{array}$ & $\begin{array}{c}0.0398 \\
{[0.1865]}\end{array}$ & $\begin{array}{c}0.0170 \\
{[0.1622]}\end{array}$ \\
\hline Popg & $\begin{array}{c}0.3983^{* * *} \\
{[0.1098]}\end{array}$ & $\begin{array}{c}0.3945^{* * *} \\
{[0.1078]}\end{array}$ & $\begin{array}{c}0.4362^{* * *} \\
{[0.1029]}\end{array}$ & $\begin{array}{c}0.4119^{* * *} \\
{[0.1043]}\end{array}$ & $\begin{array}{c}0.4002^{* * *} \\
{[0.1077]}\end{array}$ & $\begin{array}{c}0.3650^{* * *} \\
{[0.1296]}\end{array}$ \\
\hline FDI & $\begin{array}{c}0.0597 \\
{[0.0356]}\end{array}$ & $\begin{array}{c}0.0503 \\
{[0.0340]}\end{array}$ & $\begin{array}{c}0.0669^{\star} \\
{[0.0358]}\end{array}$ & $\begin{array}{l}0.0612^{\star} \\
{[0.0342]}\end{array}$ & $\begin{array}{c}0.0586 \\
{[0.0362]}\end{array}$ & $\begin{array}{l}0.0858^{* *} \\
{[0.0352]}\end{array}$ \\
\hline Trade & $\begin{array}{c}0.5929^{* * *} \\
{[0.0976]}\end{array}$ & $\begin{array}{c}0.6256^{* * *} \\
{[0.0931]}\end{array}$ & $\begin{array}{c}0.5548^{* * *} \\
{[0.1001]}\end{array}$ & $\begin{array}{c}0.6089^{* * *} \\
{[0.0972]}\end{array}$ & $\begin{array}{c}0.6133^{* * *} \\
{[0.0953]}\end{array}$ & $\begin{array}{c}0.5240^{* * *} \\
{[0.0947]}\end{array}$ \\
\hline
\end{tabular}


Continued

\begin{tabular}{ccccccc}
\hline Inst & -0.0858 & 0.0107 & 0.0430 & 0.0247 & 0.0281 & 0.0100 \\
$\mathrm{~N}$ & {$[0.0879]$} & {$[0.1053]$} & {$[0.0648]$} & {$[0.0754]$} & {$[0.0741]$} & {$[0.0735]$} \\
No. of Group & 261 & 261 & 261 & 261 & 261 & 232 \\
No. of IVs & 29 & 29 & 29 & 29 & 29 & 29 \\
AR(2) test & 0.900 & 0.907 & 0.878 & 0.888 & 0.899 & 0.838 \\
Sargan test & 0.496 & 0.508 & 0.500 & 0.492 & 0.503 & 0.130 \\
Hansen test & 0.384 & 0.397 & 0.381 & 0.396 & 0.397 & 0.665
\end{tabular}

Notes: $\left({ }^{*}\right),\left({ }^{* *}\right),\left({ }^{* * *}\right)$ are significant at $10 \%, 5 \%$, and $1 \%$ level respectively.

Table 6. Interactions and GDP per capita growth.

\begin{tabular}{|c|c|c|c|c|c|c|}
\hline $\begin{array}{l}\text { Dep: GDP per } \\
\text { capita growth }\end{array}$ & $\begin{array}{c}(19) \\
\text { Control of } \\
\text { corruption } \\
\text { (Concor) }\end{array}$ & $\begin{array}{l}\qquad(20) \\
\text { Government } \\
\text { effectiveness } \\
\text { (Goveff) }\end{array}$ & $\begin{array}{l}\text { (21) } \\
\text { Political } \\
\text { stability } \\
\text { (Politic) }\end{array}$ & $\begin{array}{c}\text { (22) } \\
\text { Regulatory } \\
\text { quality } \\
\text { (Requa) }\end{array}$ & $\begin{array}{c}\text { (23) } \\
\text { Rule of Law } \\
\text { (Rulelaw) }\end{array}$ & $\begin{array}{c}(24) \\
\text { Voice \& } \\
\text { Accountability } \\
\text { (Voice) }\end{array}$ \\
\hline L1. GDPpcg & $\begin{array}{l}0.2622^{\star * *} \\
{[0.0453]}\end{array}$ & $\begin{array}{c}0.2333^{\star * *} \\
{[0.0514}\end{array}$ & $\begin{array}{c}0.2480^{* * *} \\
{[0.0362]}\end{array}$ & $\begin{array}{l}0.2589^{* * *} \\
{[0.0317]}\end{array}$ & $\begin{array}{c}0.2797^{\star * *} \\
{[0.0434]}\end{array}$ & $\begin{array}{c}0.2204^{\star * *} \\
{[0.0499]}\end{array}$ \\
\hline Loggdppc & $\begin{array}{c}-1.4224^{\star *} \\
{[0.6062]}\end{array}$ & $\begin{array}{c}-1.2612^{\star * *} \\
{[0.4525}\end{array}$ & $\begin{array}{c}-1.4924^{\star * *} \\
{[0.3643]}\end{array}$ & $\begin{array}{c}-1.6788^{\star * *} \\
{[0.3450]}\end{array}$ & $\begin{array}{c}-1.2418^{\star \star \star} \\
{[0.3554]}\end{array}$ & $\begin{array}{c}-1.8051^{\star * *} \\
{[0.3796]}\end{array}$ \\
\hline Capg & $\begin{array}{l}0.2588^{\star * *} \\
{[0.0109]}\end{array}$ & $\begin{array}{c}0.2610^{* * *} \\
{[0.0132]}\end{array}$ & $\begin{array}{l}0.2647^{\star * *} \\
{[0.0119]}\end{array}$ & $\begin{array}{c}0.2699^{* * *} \\
{[0.0166]}\end{array}$ & $\begin{array}{c}0.2533^{\star * *} \\
{[0.0136]}\end{array}$ & $\begin{array}{l}0.2734^{\star * *} \\
{[0.0162]}\end{array}$ \\
\hline Tech & $\begin{array}{l}0.1582^{*} \\
{[0.0856]}\end{array}$ & $\begin{array}{c}0.0354 \\
{[0.0970]}\end{array}$ & $\begin{array}{l}0.2214^{* *} \\
{[0.0840]}\end{array}$ & $\begin{array}{l}0.2869^{* *} \\
{[0.1104]}\end{array}$ & $\begin{array}{l}0.1585^{\star *} \\
{[0.0714]}\end{array}$ & $\begin{array}{c}0.1597 \\
{[0.1529]}\end{array}$ \\
\hline Humcap & $\begin{array}{c}0.8868 \\
{[0.7601]}\end{array}$ & $\begin{array}{c}0.4363 \\
{[0.6042]}\end{array}$ & $\begin{array}{c}0.5348 \\
{[0.3429]}\end{array}$ & $\begin{array}{l}1.5257^{\star * *} \\
{[0.3067]}\end{array}$ & $\begin{array}{l}0.7264^{\star *} \\
{[0.3162]}\end{array}$ & $\begin{array}{l}1.1647^{\star * *} \\
{[0.2257]}\end{array}$ \\
\hline FDI & $\begin{array}{c}2.4150^{\star * *} \\
{[0.7849]}\end{array}$ & $\begin{array}{l}2.8993^{\star *} \\
{[1.3912]}\end{array}$ & $\begin{array}{l}4.1262^{* * *} \\
{[1.3319]}\end{array}$ & $\begin{array}{c}1.5589 \\
{[0.9438]}\end{array}$ & $\begin{array}{l}2.3971^{\star} \\
{[1.2419]}\end{array}$ & $\begin{array}{l}2.9059^{* *} \\
{[1.1860]}\end{array}$ \\
\hline Trade & $\begin{array}{l}2.1227^{\star * *} \\
{[0.5060]}\end{array}$ & $\begin{array}{l}2.4871^{\star * *} \\
{[0.4486]}\end{array}$ & $\begin{array}{l}2.6466^{* * *} \\
{[0.6576]}\end{array}$ & $\begin{array}{l}1.8945^{\star * \star} \\
{[0.4658]}\end{array}$ & $\begin{array}{l}1.8546^{* * *} \\
{[0.5362]}\end{array}$ & $\begin{array}{c}2.7593^{\star * *} \\
{[0.8358]}\end{array}$ \\
\hline Inst & $\begin{array}{c}17.1791 \\
{[12.5776]}\end{array}$ & $\begin{array}{c}25.8662^{* * *} \\
{[8.6664]}\end{array}$ & $\begin{array}{l}10.3405^{\star} \\
{[5.2527]}\end{array}$ & $\begin{array}{l}6.2348^{\star} \\
{[3.2789]}\end{array}$ & $\begin{array}{l}12.5733 \\
{[8.0825]}\end{array}$ & $\begin{array}{c}10.5071^{\star *} \\
{[5.0678]}\end{array}$ \\
\hline Trade $^{\star}$ Fdi & $\begin{array}{c}-0.5381^{\star * *} \\
{[0.1621]}\end{array}$ & $\begin{array}{c}-0.6552^{\star *} \\
{[0.2952]}\end{array}$ & $\begin{array}{c}-0.9948^{\star * *} \\
{[0.3156]}\end{array}$ & $\begin{array}{c}-0.3813^{\star} \\
{[0.2107]}\end{array}$ & $\begin{array}{c}-0.5386^{\star \star} \\
{[0.2592]}\end{array}$ & $\begin{array}{c}-0.6871^{\star *} \\
{[0.2768]}\end{array}$ \\
\hline Inst* Trade & $\begin{array}{l}-3.8082 \\
{[3.0576]}\end{array}$ & $\begin{array}{c}-5.8716^{\star * *} \\
{[1.9622}\end{array}$ & $\begin{array}{c}-2.6139^{\star *} \\
{[1.2645]}\end{array}$ & $\begin{array}{l}-1.3243 \\
{[0.8283]}\end{array}$ & $\begin{array}{l}-2.5758 \\
{[1.8053]}\end{array}$ & $\begin{array}{c}-2.5423^{\star \star} \\
{[1.2176]}\end{array}$ \\
\hline Inst ${ }^{\star}$ Fdi & $\begin{array}{c}-5.4798^{\star * *} \\
{[1.6192]}\end{array}$ & $\begin{array}{c}-3.0125^{\star} \\
{[1.6240]}\end{array}$ & $\begin{array}{l}-2.5881 \\
{[1.7234]}\end{array}$ & $\begin{array}{c}-1.7379^{\star} \\
{[1.0032]}\end{array}$ & $\begin{array}{c}-4.1871^{\star *} \\
{[1.5818]}\end{array}$ & $\begin{array}{l}-1.8737 \\
{[1.4711]}\end{array}$ \\
\hline Inst $^{*}$ Trade $^{\star}$ Fdi & $\begin{array}{l}1.2419^{* * *} \\
{[0.3554]}\end{array}$ & $\begin{array}{l}0.7023^{* *} \\
{[0.3428]}\end{array}$ & $\begin{array}{l}0.7637^{\star} \\
{[0.4170]}\end{array}$ & $\begin{array}{l}0.3995^{\star} \\
{[0.2027]}\end{array}$ & $\begin{array}{c}0.8991^{\star * *} \\
{[0.3246]}\end{array}$ & $\begin{array}{c}0.4821 \\
{[0.3438]}\end{array}$ \\
\hline $\mathrm{N}$ & 260 & 261 & 260 & 232 & 260 & 261 \\
\hline No. of Group & 29 & 29 & 29 & 29 & 29 & 29 \\
\hline No. of IVs & 31 & 31 & 31 & 30 & 31 & 31 \\
\hline $\mathrm{AR}(2)$ test & 0.628 & 0.731 & 0.374 & 0.882 & 0.769 & 0.542 \\
\hline Sargan test & 0.618 & 0.665 & 0.707 & 0.545 & 0.422 & 0.805 \\
\hline Hansen test & 0.763 & 0.780 & 0.845 & 0.843 & 0.721 & 0.944 \\
\hline
\end{tabular}

Notes: $\left(^{*}\right),\left({ }^{*}\right),\left({ }^{* *}\right)$ are significant at $10 \%, 5 \%$, and $1 \%$ level respectively. 
Table 7. Interactions and GDP growth.

\begin{tabular}{|c|c|c|c|c|c|c|}
\hline $\begin{array}{l}\text { Dep: GDP } \\
\text { growth }\end{array}$ & $\begin{array}{l}(25) \\
\text { Control of } \\
\text { corruption } \\
\text { (Concor) }\end{array}$ & $\begin{array}{c}\text { (26) } \\
\text { Government } \\
\text { effectiveness } \\
\text { (Goveff) }\end{array}$ & $\begin{array}{c}(27) \\
\text { Political } \\
\text { stability } \\
\text { (Politic) }\end{array}$ & $\begin{array}{c}\text { (28) } \\
\text { Regulatory } \\
\text { quality } \\
\text { (Requa) }\end{array}$ & $\begin{array}{c}\text { (29) } \\
\text { Rule of Law } \\
\text { (Rulelaw) }\end{array}$ & $\begin{array}{c}(30) \\
\text { Voice \& } \\
\text { Accountability } \\
\text { (Voice) }\end{array}$ \\
\hline L1. GDPg & $\begin{array}{c}0.2486^{* * *} \\
{[0.0462]}\end{array}$ & $\begin{array}{c}0.2625^{\star * *} \\
{[0.0423]}\end{array}$ & $\begin{array}{c}0.2226^{* * *} \\
{[0.0479]}\end{array}$ & $\begin{array}{c}0.2010^{* * *} \\
{[0.0526]}\end{array}$ & $\begin{array}{c}0.2291^{\star * *} \\
{[0.0462]}\end{array}$ & $\begin{array}{c}0.2419 \\
{[0.0449]}\end{array}$ \\
\hline Loggdppc & $\begin{array}{c}-0.8526^{*} \\
{[0.4963]}\end{array}$ & $\begin{array}{c}-1.4012^{* * *} \\
{[0.4439]}\end{array}$ & $\begin{array}{c}-1.1187^{\star} \\
{[0.5744]}\end{array}$ & $\begin{array}{c}-1.5300^{* * *} \\
{[0.3998]}\end{array}$ & $\begin{array}{c}-1.0924^{* * *} \\
{[0.3331]}\end{array}$ & $\begin{array}{l}-1.3661 \\
{[0.4123]}\end{array}$ \\
\hline Capg & $\begin{array}{c}0.2410^{* * *} \\
{[0.0106]}\end{array}$ & $\begin{array}{c}0.2400^{* * *} \\
{[0.0097]}\end{array}$ & $\begin{array}{c}0.2550^{* * *} \\
{[0.0106]}\end{array}$ & $\begin{array}{c}0.2523^{* * *} \\
{[0.0142]}\end{array}$ & $\begin{array}{c}0.2423^{* * *} \\
{[0.0108]}\end{array}$ & $\begin{array}{c}0.2518 \\
{[0.0132]}\end{array}$ \\
\hline Tech & $\begin{array}{c}0.1934^{* * *} \\
{[0.0639]}\end{array}$ & $\begin{array}{c}0.1567^{* * *} \\
{[0.0521]}\end{array}$ & $\begin{array}{c}0.2751^{\star * *} \\
{[0.0920]}\end{array}$ & $\begin{array}{c}0.2291^{\star * *} \\
{[0.0820]}\end{array}$ & $\begin{array}{l}0.1759^{\star *} \\
{[0.0644]}\end{array}$ & $\begin{array}{c}0.1782 \\
{[0.0963]}\end{array}$ \\
\hline Humcap & $\begin{array}{c}0.0667 \\
{[0.7282]}\end{array}$ & $\begin{array}{c}0.5417 \\
{[0.5589]}\end{array}$ & $\begin{array}{c}0.3392 \\
{[0.9711]}\end{array}$ & $\begin{array}{c}1.0353^{\star} \\
{[0.5431]}\end{array}$ & $\begin{array}{c}0.4188 \\
{[0.4742]}\end{array}$ & $\begin{array}{c}0.8041 \\
{[0.4948]}\end{array}$ \\
\hline Popg & $\begin{array}{l}0.4079^{* *} \\
{[0.1956]}\end{array}$ & $\begin{array}{l}0.2195^{* *} \\
{[0.0859]}\end{array}$ & $\begin{array}{c}0.2443 \\
{[0.1887]}\end{array}$ & $\begin{array}{c}0.4187^{\star} \\
{[0.2359]}\end{array}$ & $\begin{array}{c}0.4895^{\star} \\
{[0.2435]}\end{array}$ & $\begin{array}{c}0.4360 \\
{[0.2466]}\end{array}$ \\
\hline FDI & $\begin{array}{l}2.1709^{* *} \\
{[0.8703]}\end{array}$ & $\begin{array}{l}3.4587^{\star *} \\
{[1.2694]}\end{array}$ & $\begin{array}{c}1.6079 \\
{[1.0958]}\end{array}$ & $\begin{array}{c}2.8189^{* * *} \\
{[0.9326]}\end{array}$ & $\begin{array}{c}2.1747^{\star * *} \\
{[0.6599]}\end{array}$ & $\begin{array}{c}3.0573 \\
{[1.3057]}\end{array}$ \\
\hline Trade & $\begin{array}{c}1.6511^{* * *} \\
{[0.3780]}\end{array}$ & $\begin{array}{c}2.3589^{* * *} \\
{[0.4328]}\end{array}$ & $\begin{array}{c}1.8513^{* * *} \\
{[0.5957]}\end{array}$ & $\begin{array}{c}2.1147^{* * *} \\
{[0.5443]}\end{array}$ & $\begin{array}{c}1.8476^{* * *} \\
{[0.3168]}\end{array}$ & $\begin{array}{c}1.9541 \\
{[0.6773]}\end{array}$ \\
\hline Inst & $\begin{array}{c}19.2223 \\
{[14.3523]}\end{array}$ & $\begin{array}{l}10.8902^{*} \\
{[6.3954]}\end{array}$ & $\begin{array}{c}3.6832 \\
{[8.2781]}\end{array}$ & $\begin{array}{c}2.4483 \\
{[3.6222]}\end{array}$ & $\begin{array}{l}10.8101 \\
{[7.0245]}\end{array}$ & $\begin{array}{c}5.0460 \\
{[4.4149]}\end{array}$ \\
\hline Trade Fdi & $\begin{array}{c}-0.4749^{* *} \\
{[0.1886]}\end{array}$ & $\begin{array}{c}-0.7678^{\star} \\
{[0.2737]}\end{array}$ & $\begin{array}{l}-0.3812 \\
{[0.2574]}\end{array}$ & $\begin{array}{c}-0.6377^{* * *} \\
{[0.1961]}\end{array}$ & $\begin{array}{c}-0.4807^{* * *} \\
{[0.1378]}\end{array}$ & $\begin{array}{l}-0.6559 \\
{[0.2862]}\end{array}$ \\
\hline Inst ${ }^{\star}$ Trade & $\begin{array}{l}-4.5542 \\
{[3.4630]}\end{array}$ & $\begin{array}{l}-2.3409 \\
{[1.4330]}\end{array}$ & $\begin{array}{l}-0.8113 \\
{[1.9992]}\end{array}$ & $\begin{array}{l}-0.5867 \\
{[0.8620]}\end{array}$ & $\begin{array}{l}-2.2846 \\
{[1.4802]}\end{array}$ & $\begin{array}{l}-0.9764 \\
{[1.0487]}\end{array}$ \\
\hline Inst ${ }^{\star}$ Fdi & $\begin{array}{c}-3.9483^{*} \\
{[2.2542]}\end{array}$ & $\begin{array}{c}-4.2323^{* * *} \\
{[1.2988]}\end{array}$ & $\begin{array}{c}-2.6174^{* *} \\
{[1.2795]}\end{array}$ & $\begin{array}{l}-1.1435 \\
{[0.8396]}\end{array}$ & $\begin{array}{c}-3.6231^{*} \\
{[2.0299]}\end{array}$ & $\begin{array}{l}-3.6142 \\
{[1.4751]}\end{array}$ \\
\hline Inst $^{\star}$ Trade $^{\star}$ Fdi & $\begin{array}{c}0.9398^{*} \\
{[0.5107]}\end{array}$ & $\begin{array}{c}0.9615^{* * *} \\
{[0.2878]}\end{array}$ & $\begin{array}{c}0.6174^{*} \\
{[0.3140]}\end{array}$ & $\begin{array}{c}0.2981^{*} \\
{[0.1748]}\end{array}$ & $\begin{array}{c}0.7888^{*} \\
{[0.4072]}\end{array}$ & $\begin{array}{c}0.7680 \\
{[0.3082]}\end{array}$ \\
\hline $\mathrm{N}$ & 261 & 261 & 260 & 233 & 261 & 261 \\
\hline No. of Group & 29 & 29 & 29 & 29 & 29 & 29 \\
\hline No. of IVs & 33 & 33 & 33 & 31 & 33 & 31 \\
\hline $\operatorname{AR}(2)$ test & 0.422 & 0.652 & 0.679 & 0.911 & 0.652 & 0.542 \\
\hline Sargan test & 0.335 & 0.319 & 0.452 & 0.142 & 0.233 & 0.805 \\
\hline Hansen test & 0.817 & 0.873 & 0.740 & 0.820 & 0.835 & 0.944 \\
\hline
\end{tabular}

Notes: $\left(^{*}\right),\left({ }^{* *}\right),\left({ }^{* *}\right)$ are significant at $10 \%, 5 \%$, and $1 \%$ level respectively.

and Trade respectively on economic growth via interaction terms. While Trade and FDI show the expected positive impacts on economic growth, the interactions between institutional quality and trade openness (Trade)/financial openness (FDI) have significant negative coefficients. These results indicate that the improvement in institutional quality will have less positive impacts on economic growth in the case of more opened trade account. This confirms hypothesis 1 . The increasing trade openness of emerging markets invites competition from foreign firms especially from developed countries, and institutional quality improvement will further strengthen the competition, leading to economic slowdown in the short run. This result is in line with findings from African countries 
[38] but contradicts the findings from developed economies [14]. This can be explained by the fact that the competitive capability of emerging markets are low so that foreign firms have more advantages in competing with the domestic firms when the institutional quality is improved thus the economic growth is lower.

The coefficient of the interaction between institutions and FDI is negative. This does not support hypothesis 2 and contradicts the findings of [17] that better institutional quality can promote the growth effect of FDI. Our results show that the growth effect of FDI is stronger in countries with lower institutional quality. This controversial result can be explained that FDI may provide greater incentive for institutions improvement in countries with low institutional quality, which in turn positively supports economic growth.

Interestingly, we find that the interaction between FDI and Trade has a negative impact on economic growth. We posit that trade openness brings fierce international competition to emerging markets [14], faces domestic firms to unfavorable environment, and hence, impedes the FDI spillover effect in the short run. However, the negative effect of trade in association with FDI is lessened in the countries with high institutional quality. This is reflected in the significant positive coefficient of the nexus of institutional quality, trade openness and FDI. This novel result indicates that institutional quality improvement can mitigate the competition brought by trade openness in the areas that FDIs operate to optimize their spillover effect.

\section{Conclusion}

Institutional quality has an important role in stimulating economic activities and accelerating economic growth especially in emerging markets where great efforts have been put to improve institutional quality. In this paper, we collect data in the period of 2002-2015 for 29 emerging markets to examine the impacts of institutional quality on economic growth. By conducting the system-GMM estimators, we find that the institutional quality has positive impacts on economic growth in emerging markets. In addition, institutional quality improvement has negative impacts on the economic growth effect of trade openness and FDI. The findings also demonstrate that the competition brought by trade openness may impede the spill-over effect of FDI. However, better institutional can mitigate the competition brought by trade openness in the areas that FDIs operate to optimize their spill-over effect. Policy makers in emerging economies should improve institutional quality and competitive capacity of domestic firms before promoting trade openness and FDI. This also provides implication to trade policies for the period 2015-2018 that emerging economies should focus on improving their capability in absorbing the benefits from economic integration with stronger institutional reforms.

\section{Acknowledgements}

This study is funded by University of Economics Ho Chi Minh City, Vietnam. 


\section{References}

[1] Poshakwale, S. and Ganguly, G. (2015) International Shocks and Growth in Emerging Markets. Global Finance Journal, 26, 29-46. https://doi.org/10.1016/j.gfj.2015.01.003

[2] Auzina-Emsina, A. (2014) Labour Productivity, Economic Growth and Global Competitiveness in Post-Crisis Period. Procedia-Social and Behavioral Sciences, 156, 317-321. https://doi.org/10.1016/j.sbspro.2014.11.195

[3] Demeter, K., Chikán, A. and Matyusz, Z. (2011) Labour Productivity Change: Drivers, Business Impact and Macroeconomic Moderators. International Journal of Production Economics, 131, 215-223. https://doi.org/10.1016/j.ijpe.2010.11.003

[4] Giordano, C. and Giugliano, F. (2015) A Tale of Two Fascisms: Labour Productivity Growth and Competition Policy in Italy, 1911-1951. Explorations in Economic History, 55, 25-38. https://doi.org/10.1016/j.eeh.2013.12.003

[5] Kurt, S. and Kurt, Ü. (2015) Innovation and Labour Productivity in BRICS Countries: Panel Causality and Co-Integration. Procedia-Social and Behavioral Sciences, 195, 1295-1302. https://doi.org/10.1016/j.sbspro.2015.06.296

[6] Almeida, F. (2015) The Psychology of Early Institutional Economics: The Instinctive Approach of Thorstein Veblen's Conspicuous Consumer Theory. Economia, 16, 226-334. https://doi.org/10.1016/j.econ.2015.05.002

[7] Chan, S.-G., Koh, E.H.Y., Zainir, F. and Yong, C.-C. (2015) Market Structure, Institutional Framework and Bank Efficiency in ASEAN 5. Journal of Economics and Business, 82, 84-112. https://doi.org/10.1016/j.jeconbus.2015.07.002

[8] Djankov, S., La Porta, R., Lopez-de-Silanes, F. and Shleifer, A. (2002) The Regulation of Entry. Quarterly Journal of Economics, 117, 1-37.

https://doi.org/10.1162/003355302753399436

[9] Zhang, S. (2016) Institutional Arrangements and Debt Financing. Research in International Business and Finance, 36, 362-372. https://doi.org/10.1016/j.ribaf.2015.10.006

[10] Dal Bó, E. and Rossi, M.A. (2007) Corruption and Inefficiency: Theory and Evidence from Electric Utilities. Journal of Public Economics, 91, 939-962. https://doi.org/10.1016/j.jpubeco.2006.11.005

[11] Park, J. (2012) Corruption, Soundness of the Banking Sector, and Economic Growth: A Cross-Country Study. Journal of International Money and Finance, 31, 907-929. https://doi.org/10.1016/j.jimonfin.2011.07.007

[12] Lucifora, C. and Moriconi, S. (2015) Political Instability and Labour Market Institutions. European Journal of Political Economy, 39, 201-221.

https://doi.org/10.1016/j.ejpoleco.2015.05.003

[13] Farhadi, M., Islam, M.R. and Moslehi, S. (2015) Economic Freedom and Productivity Growth in Resource-Rich Economies. World Development, 72, 109-126. https://doi.org/10.1016/j.worlddev.2015.02.014

[14] Hadhek, Z. and Mrad, F. (2015) Trade Openness, Institutions and Economic Growth. European Journal of Economics, Finance and Administrative Sciences, No. 75, 96-104.

[15] Pegkas, P. (2015) The Impact of FDI on Economic Growth in Eurozone Countries. The Journal of Economic Asymmetries, 12, 124-132. https://doi.org/10.1016/j.jeca.2015.05.001

[16] Dollar, D. and Kraay, A. (2003) Institutions, Trade, and Growth. Journal of Mone- 
tary Economics, 50, 133-162. https://doi.org/10.1016/S0304-3932(02)00206-4

[17] Jude, C. and Levieuge, G. (2015) Growth Effect of FDI in Developing Economies: The Role of Institutional Quality. LEO Working Papers/DR LEO, 2251.

[18] Rivera-Batiz, L.A. and Romer, P.M. (1991) Economic Integration and Endogenous Growth. The Quarterly Journal of Economics, 106, 531-555. https://doi.org/10.2307/2937946

[19] Rivera-Batiz, L.A. and Romer, P.M. (1991) International Trade with Endogenous Technological Change. European Economic Review, 35, 971-1001. https://doi.org/10.1016/0014-2921(91)90048-N

[20] Yanikkaya, H. (2003) Trade Openness and Economic Growth: A Cross-Country Empirical Investigation. Journal of Development Economics, 72, 57-89. https://doi.org/10.1016/S0304-3878(03)00068-3

[21] Anderson, J.E. and Marcouiller, D. (2002) Insecurity and the Pattern of Trade: An Empirical Investigation. Review of Economics and Statistics, 84, 342-352. https://doi.org/10.1162/003465302317411587

[22] Yu, M. (2010) Trade, Democracy, and the Gravity Equation. Journal of Development Economics, 91, 289-300. https://doi.org/10.1016/j.jdeveco.2009.07.004

[23] Peter, K.S., Svejnar, J. and Terrell, K. (2012) Foreign Investment, Corporate Ownership, and Development: Are Firms in Emerging Markets Catching up to the World Standard? Review of Economics and Statistics, 94, 981-991. https://doi.org/10.1162/REST_a_00315

[24] Grossman, G.M. and Helpman, E. (1991) Quality Ladders in the Theory of Growth. The Review of Economic Studies, 58, 43-61. https://doi.org/10.2307/2298044

[25] Barro, R.J. and Sala-i-Martin, X. (1995) Technological Diffusion, Convergence, and Growth. National Bureau of Economic Research. https://doi.org/10.3386/w5151

[26] Mody, A. and Murshid, A.P. (2005) Growing up with Capital Flows. Journal of International Economics, 65, 249-266. https://doi.org/10.1016/j.jinteco.2004.02.003

[27] Lee, J.-W. and Hong, K. (2012) Economic Growth in Asia: Determinants and Prospects. Japan and the World Economy, 24, 101-113. https://doi.org/10.1016/j.japwor.2012.01.005

[28] Bhattacharya, M., Rafiq, S. and Bhattacharya, S. (2015) The Role of Technology on the Dynamics of Coal Consumption-Economic Growth: New Evidence from China. Applied Energy, 154, 686-695. https://doi.org/10.1016/j.apenergy.2015.05.063

[29] Ceccobelli, M., Gitto, S. and Mancuso, P. (2012) ICT Capital and Labour Productivity Growth: A Non-Parametric Analysis of 14 OECD Countries. Telecommunications Policy, 36, 282-292. https://doi.org/10.1016/j.telpol.2011.12.012

[30] Omri, A. (2014) The Nexus among Foreign Investment, Domestic Capital and Economic Growth: Empirical Evidence from the MENA Region. Research in Economics, 68, 257-263. https://doi.org/10.1016/j.rie.2013.11.001

[31] Herrera-Echeverri, H., Haar, J. and Estévez-Bretón, J.B. (2014) Foreign Direct Investment, Institutional Quality, Economic Freedom and Entrepreneurship in Emerging Markets. Journal of Business Research, 67, 1921-1932. https://doi.org/10.1016/j.jbusres.2013.11.020

[32] Blundell, R. and Bond, S. (1998) Initial Conditions and Moment Restrictions in Dynamic Panel Data Models. Journal of Econometrics, 87, 115-143. https://doi.org/10.1016/S0304-4076(98)00009-8

[33] Blundell, R., Bond, S. and Windmeijer, F. (2001) Estimation in Dynamic Panel Data Models: Improving on the Performance of the Standard GMM Estimator. Nonsta- 
tionary Panels, Panel Cointegration, and Dynamic Panels: Emerald Group Publishing Limited, 53-91.

[34] Herrmann, S. and Winkler, A. (2009) Real Convergence, Financial Markets, and the Current Account-Emerging Europe versus Emerging Asia. The North American Journal of Economics and Finance, 20, 100-123.

https://doi.org/10.1016/j.najef.2009.05.001

[35] Fung, M.K. (2009) Financial Development and Economic Growth: Convergence or Divergence? Journal of International Money and Finance, 28, 56-67.

https://doi.org/10.1016/j.jimonfin.2008.08.001

[36] Young, A.T. and Sheehan, K.M. (2014) Foreign Aid, Institutional Quality, and Growth. European Journal of Political Economy, 36, 195-208.

https://doi.org/10.1016/j.ejpoleco.2014.08.003

[37] Wang, Y., Cheng, L., Wang, H. and Li, L. (2014) Institutional Quality, Financial Development and OFDI. Pacific Science Review, 16, 127-132. https://doi.org/10.1016/j.pscr.2014.08.023

[38] Baliamoune, M. and Ndikumana, L. (2007) The Growth Effects of Openness to Trade and the Role of Institutions: New Evidence from African Countries. University Library of Munich, Munich. 


\section{Appendices}

Appendix 1. List of emerging countries in the sample.

\begin{tabular}{ccccc}
\hline Argentina & Colombia & Indonesia & Pakistan & Slovenia \\
\hline Bangladesh & Czech Republic & Iran, Islamic Rep. & Peru & Thailand \\
Brazil & Egypt, Arab Rep. & Israel & Philippines & Turkey \\
Bulgaria & Greece & Korea, Rep. & Poland & Ukraine \\
Chile & Hungary & Malaysia & Romania & Vietnam \\
China & India & Mexico & Russian Federation \\
\hline
\end{tabular}

Note: the list of emerging economies is followed the classification of MSCI

(https://www.msci.com/emerging-markets) in combining with other sources as IMF (www.imf.org/external/pubs/ft/weo/2015/02/pdf/text.pdf).

Appendix 2. Variable definitions.

\begin{tabular}{|c|c|c|c|}
\hline Variables & & Definitions & Sources \\
\hline \multirow[t]{9}{*}{ Dependent var. } & Gdpg & Real GDP growth rate (annual, \%) & WDI \\
\hline & Gdppcg & Real GDP per capita growth rate (annual, \%) & WDI \\
\hline & & Control variables & \\
\hline & Capg & Gross capital formation (annual \% growth) & WDI \\
\hline & Tech & $\begin{array}{l}\text { Logarithms of total nonresident and resident patent } \\
\text { applications }\end{array}$ & WDI \\
\hline & Loggdppc & Logarithms of real GDP per capita & WDI \\
\hline & Popg & Population growth (annual \%) & WDI \\
\hline & Humcap & $\begin{array}{l}\text { Logarithms of ratio of tertiary school enrollment to } \\
\text { population }\end{array}$ & WDI \\
\hline & & Explanatory variables & \\
\hline \multirow{5}{*}{$\begin{array}{l}\text { Economic } \\
\text { openness }\end{array}$} & Trade & $\begin{array}{l}\text { Logarithms of ratio trade to GDP (trade is total value of } \\
\text { exports and imports) }\end{array}$ & WDI \\
\hline & Fdi & Foreign direct investment, net inflows (\% of GDP) & WDI \\
\hline & Concor & Control of Corruption indicator (estimated number) & WGI \\
\hline & Goveff & Government effectiveness indicator (estimated number) & WGI \\
\hline & Politic & $\begin{array}{l}\text { Political Stability and Absence of Violence/Terrorism } \\
\text { (estimated number) }\end{array}$ & WGI \\
\hline & Requa & Regulatory quality (estimated number) & WGI \\
\hline & Rulelaw & Rule of law (estimated number) & WGI \\
\hline & Voice & Voice and Accountability (estimated number) & WGI \\
\hline
\end{tabular}

